\title{
Ensino de gestão em saúde nos cursos paranaenses de Odontologia
}

\author{
Najara Barbosa da Rocha*; Ieda Harumi Higarashi** \\ * Professor Adjunto, Departamento de Odontologia Social e \\ Preventiva, Faculdade de Odontologia, UFMG \\ ** Professora Associada, Departamento de Enfermagem, UEM
}

Recebido em 15/05/2019. Aprovado em 30/07/2019.

\begin{abstract}
RESUMO
Objetivou-se analisar o ensino da gestão na Odontologia das universidades do estado do Paraná. Trata-se de pesquisa descritiva, transversal, a partir de dados secundários, no ano de 2019. Foram coletadas informações das instituições de ensino superior, públicas e privadas, que ofereciam curso de Odontologia, tendo como referência a lista de cursos aprovados, iniciados e ativos do cadastro nacional de cursos de educação superior (Cadastro e-MEC). A síntese dos dados foi realizada em formulário padronizado no programa Microsoft Excel, que após a digitação, permitiu análise pelo programa EpiInfo (Versão 7.2) de forma descritiva, e apresentação por meio de frequências absoluta e relativa. Análises bivariadas foram realizadas pelo programa Bioestat utilizando o teste $\mathrm{G}$ e Exato de Fischer, ao nível de significância de 5\%. No Paraná são ofertados 29 cursos de Odontologia, sendo 5 aprovados, porém ainda não iniciaram suas atividades. Dos cursos pesquisados, 21 são de natureza administrativa privada (particular), sete são públicos e apenas um é público, porém cobra mensalidade dos alunos da graduação. A maioria dos cursos $(58,6 \%)$ tem disciplina específica de gestão, como componente curricular obrigatório $(96,6 \%)$, ofertada predominantemente no último ano de graduação $(55,2 \%)$, com carga horária variando entre 21 e 60 horas-aula (61.9\%). A maioria $(51,7 \%)$ dos cursos de graduação em Odontologia dedica apenas até 1\% da carga horária para gestão em saúde. Os resultados mostraram que o ensino de gestão neste estado é pouco explorado, fazendo-se necessária inclusão de maior carga horária voltada para o tema nas matrizes curriculares, objetivando melhorar a formação do futuro cirurgião-dentista.
\end{abstract}

Descritores: Educação Superior. Educação em Odontologia. Gestão em Saúde. Odontologia.

\section{INTRODUÇÃO}

O perfil do cirurgião-dentista, apontado pelas diretrizes curriculares nacionais (DCN) para os cursos de Odontologia (2002) descreve um profissional com conhecimentos, habilidades e competências que permitam decidir e atuar com segurança e propriedade na promoção da saúde e na prevenção para atender às necessidades sociais, 
mas que não seja um "operário da Odontologia", com mentalidade puramente tecnicista. Em outras palavras, almeja-se um profissional que consiga interagir com a sociedade e que tenha capacidade de liderança e sensibilidade social, com vasta vivência clínica, com técnicas sofisticadas de cura sustentada por uma sólida base em ciências básicas, que possa exercer a profissão em consultório privado, mas que se adapte a equipes multidisciplinares e serviços socializados. Uma das competências apontadas pelas DCN é a capacidade de gerenciar e administrar tanto a força de trabalho, quanto os recursos físicos, materiais e de informação, ou seja, os egressos devem estar aptos a atuar como gestores, empregadores ou lideranças na equipe de saúde ${ }^{1}$.

$\mathrm{Na}$ área da saúde, a formação de novos profissionais tem sido concebida de forma linear, ou seja, não ocorre o diálogo necessário entre as diferentes áreas do conhecimento. Tal concepção de formação produz pessoas com uma visão limitada do que é sua área de atuação ${ }^{2}$.

O processo de gestão apóia a viabilização da prestação do atendimento ao paciente e envolve questões relacionadas à sua qualidade. Sendo assim, esses profissionais, uma vez graduados, necessitarão comportarem-se como gestores, e, portanto, desenvolver uma visão de mercado consumidor e habilidade para administração. Um mau gestor prejudica seus clientes, colaboradores e sociedade, pois intervém de forma equivocada em aspectos que impactam a qualidade de vida dos agentes organizacionais ${ }^{2}$.

A formação dos profissionais da área de saúde deveria ser concebida por meio do quadrilátero ensino, gestão, atenção e controle social. Essa concepção multidimensional possibilita que a área da saúde progrida qualitativamente no atendimento da população. Para transformar a organização de serviços e dos processos formativos, é necessário um trabalho articulado entre o sistema de saúde (em suas várias esferas de gestão) e as instituições formadoras. É necessário que, na dimensão do ensino, se considerem aspectos relacionados à administração em si, no contexto da gestão pública, e com a atenção voltada ao atendimento e ao controle social, reconhecendo o envolvimento da população no processo de concepção da saúde. Esta proposta proporcionará ao profissional da saúde uma visão mais integradora do campo de atuação, possibilitando uma ação mais competente ${ }^{3}$.

A barreira na área de saúde, envolvendo o processo de formação e a gestão de organizações, precisa ser estudado, para que a formação do profissional de saúde seja repensada, visto que tanto no setor público como no privado, o profissional da Odontologia é exposto a atividades que necessitam deste conhecimento, e ainda, que sua atuação em algumas situações é fragilizada pela ausência desse tipo de formação, prejudicando não apenas seu desempenho, como também a comunidade em que está inserido. Assim, este estudo teve como objetivo analisar o ensino da gestão nos cursos de Odontologia do Estado do Paraná.

\section{METODOLOGIA}

Trata-se de uma pesquisa descritiva, transversal, a partir de dados secundários, sobre o ensino de gestão na Odontologia no estado do Paraná, em fevereiro de 2019.

Foram coletadas informações das instituições de ensino superior do estado, públicas e privadas, que ofereciam curso de Odontologia, tendo como referência a lista de cursos aprovados, iniciados e ativos do cadastro nacional de cursos e instituições de educação superior. O Cadastro eMEC, regulamentado pela Portaria Normativa $n^{\circ}$ 21, de 21/12/2017, constitui uma base de dados oficial dos cursos e Instituições de Educação Superior (IES), independentemente de Sistema de Ensino. Os dados do Cadastro e-MEC devem guardar conformidade com os atos autorizativos 
dos cursos e das IES, editados pelo Poder Público ou órgão competente das instituições, de acordo com o exercício de sua autonomia ${ }^{4}$. Foram encontrados 29 cursos de Odontologia no estado do Paraná, excluindo 5 cursos que ainda não haviam sido iniciados até 2019.

De posse do cadastro e dados de todas as IES, uma pesquisadora treinada e calibrada acessou o sítio web de cada instituição. Foram analisadas as matrizes curriculares dos cursos de Odontologia e ementas, sendo observada a presença de componentes curriculares que abordassem o ensino de gestão. Quando as informações não eram encontradas por meio desta sistemática, era realizada uma pesquisa no Google com as seguintes palavras-chave: matriz curricular, Odontologia e o nome da instituição.

Foram analisadas as seguintes variáveis: natureza administrativa (instituição pública ou privada); se possui a disciplina de gestão ou alguma disciplina que abordasse o assunto, nomenclatura da disciplina, carga horária e momento na graduação em que a disciplina é oferecida, natureza do componente curricular (obrigatória ou optativa); nota Enade do curso, carga horária do curso de Odontologia e o valor da mensalidade, se aplicável. Algumas variáveis como natureza da IES, nota Enade e carga horária do curso foram associados com a variável desfecho, presença de disciplina de gestão no currículo do curso.

A síntese dos dados foi realizada em um formulário padronizado no programa Microsoft Excel, seguida de análise descritiva, com apresentação dos dados por meio de frequências absoluta e relativa (EpiInfo 7.2) ${ }^{5}$. A análise estatística foi realizada no programa Bioestat $5.0^{6} \mathrm{e}$ utilizou os testes Exato de Fischer ou $G$ de Willians, considerando o intervalo de confiança de 95\% e nível de significância de 5\%.

\section{RESULTADOS}

Do total de cursos (n=29), 21 são de natureza administrativa privada (particular), sete são públicos e uma universidade é fundação municipal e cobra mensalidades dos alunos da graduação a partir do ano de 2019. De acordo com a tabela 1, a maioria das IES oferece curso integral $(65,6 \%)$, integralizados em 5 anos (41,4\%), tem estrutura semestral (55,3\%), sem avaliação no Enade (43,5\%), com carga horária de 4001 a 4500 horas $(48,3 \%)$ e cobra mensalidade $\mathrm{R} \$ 2.501,00$ a $\mathrm{R} \$$ $3.000,00(27,6 \%)$.

As nomenclaturas dos componentes curriculares encontrados que contemplam conteúdos de gestão foram: Empreendedorismo, Administração aplicada à Odontologia; Estágio de Saúde Coletiva - Gestão do SUS; Gestão de serviços públicos de Odontologia; Gestão e Planejamento em Odontologia; Gestão pública e privada em Odontologia; Orientação profissional; Políticas públicas, planejamento e gestão em saúde; Atitude empreendedora. Também foi encontrado o ensino da gestão em disciplinas não específicas como Odontologia Social e Preventiva.

Segundo a tabela 2, no que diz respeito à acessibilidade às informações, a maioria dos cursos de Odontologia permite fácil acesso pela internet $(96,6 \%)$ e disponibiliza a matriz curricular $(82,8 \%)$. Entretanto, a maioria não oferece acesso à matriz e ementas das disciplinas de forma detalhada $(72,4 \%)$. Verifica-se que a maioria dos cursos $(58,6 \%)$ tem disciplina específica de gestão, com componente curricular de natureza obrigatória (96,6\%). Em um dos casos é oferecida na forma de disciplina de ensino à distância e, em outro, sob formato de disciplina optativa. Estes componentes curriculares são ofertados predominantemente no último ano de graduação (55,2\%), com carga horária variando entre 21 e 60 horas-aula (61.9\%).

Ao verificar-se a carga horária destinada ao conteúdo/disciplina de gestão, em relação à carga horária total do curso, constata-se a dedicação de um número bastante reduzido de horas à abordagem deste conteúdo. A maioria $(51,7 \%)$ dos 
cursos de graduação em Odontologia dedica até $1 \%$ da carga horária do curso para a gestão em saúde (tabela 2).

Nas associações da presença de disciplina específica sobre gestão e carga horária do curso de graduação, natureza administrativa e nota do Enade, nenhuma das variáveis se mostrou significativa, como demonstra a tabela 3 .

Tabela 1. Distribuição percentual e numérica das características dos cursos de Odontologia do Estado do Paraná, 2019

\begin{tabular}{|c|c|c|}
\hline Características dos cursos & $\mathbf{n}$ & $\%$ \\
\hline \multicolumn{3}{|l|}{ Natureza administrativa } \\
\hline Particular & 21 & 72,5 \\
\hline Público & 7 & 24,1 \\
\hline Fundação Municipal com mensalidade & 1 & 3,4 \\
\hline \multicolumn{3}{|l|}{ Turno de funcionamento } \\
\hline Integral diurno e noturno & 9 & 31,0 \\
\hline Somente integral diurno & 19 & 65,6 \\
\hline Somente noturno & 1 & 3,4 \\
\hline \multicolumn{3}{|l|}{ Duração em anos } \\
\hline 4 & 9 & 31,0 \\
\hline 4,5 & 3 & 10,4 \\
\hline 5 & 12 & 41,4 \\
\hline Variável de acordo com o turno* & 5 & 17,2 \\
\hline \multicolumn{3}{|l|}{ Estrutura/Periodização } \\
\hline Anual & 12 & 41,3 \\
\hline Semestral & 16 & 55,3 \\
\hline Anual e semestral & 1 & 3,4 \\
\hline \multicolumn{3}{|l|}{ Nota no Enade } \\
\hline Sem avaliação & 10 & 34,5 \\
\hline 2 & 6 & 20,7 \\
\hline 3 & 5 & 17,2 \\
\hline 4 & 6 & 20,7 \\
\hline 5 & 2 & 6,9 \\
\hline \multicolumn{3}{|l|}{ Carga horária (horas-aula) } \\
\hline Até 4000 & 5 & 17,2 \\
\hline 4001 a 4500 & 14 & 48,3 \\
\hline 4501 a 5000 & 8 & 27,6 \\
\hline Mais de 5000 & 2 & 6,9 \\
\hline \multicolumn{3}{|l|}{ Valor da mensalidade } \\
\hline Gratuito (público) & 7 & 24,2 \\
\hline Não informado & 3 & 10,3 \\
\hline Até $\mathrm{R} \$ 2.000,00$ & 2 & 6,9 \\
\hline De $R \$ 2.001,00$ a $R \$ 2.500,00$ & 5 & 17,2 \\
\hline De $R \$ 2.501,00$ a $R \$ 3.000,00$ & 8 & 27,6 \\
\hline Mais de $\mathrm{R} \$ 3.000,00$ & 4 & 13,8 \\
\hline
\end{tabular}

*4 anos para o período integral e 5 anos para o noturno. 
Tabela 2. Distribuição numérica e percentual dos cursos, de acordo com o acesso às suas informações, matriz curricular e ementas, 2019

\begin{tabular}{|c|c|c|}
\hline Variáveis & $\mathbf{n}$ & $\%$ \\
\hline \multicolumn{3}{|l|}{ Facilidade de acesso ao sítio web } \\
\hline Sim & 28 & 96,6 \\
\hline Não & 1 & 3,4 \\
\hline \multicolumn{3}{|l|}{ Facilidade de acesso à matriz } \\
\hline Sim & 24 & 82,8 \\
\hline Não & 5 & 17,2 \\
\hline \multicolumn{3}{|l|}{ Disponibilidade de matriz curricular detalhada } \\
\hline Sim & 8 & 27,6 \\
\hline Não & 18 & 62,1 \\
\hline Não apresenta matriz no sítio $w e b$ & 3 & 10,3 \\
\hline \multicolumn{3}{|l|}{ Existência de disciplina específica de gestão } \\
\hline Sim & 16 & 55,3 \\
\hline Não & 8 & 27,6 \\
\hline Não apresenta matriz no sítio web & 3 & 10,3 \\
\hline Não apresenta a disciplina de gestão na matriz & 1 & 3,4 \\
\hline Disciplina optativa & 1 & 3,4 \\
\hline \multicolumn{3}{|l|}{ Carga horária dedicada à gestão (horas-aula) } \\
\hline Até 20 & 1 & 3,4 \\
\hline 21 a 60 & 13 & 44,7 \\
\hline 61 a 100 & 6 & 20,8 \\
\hline Mais de 100 & 3 & 10,3 \\
\hline Não tem horas ou não informado & 6 & 20,8 \\
\hline \multicolumn{3}{|l|}{ Ano da graduação em que se insere a disciplina/conteúdo de gestão } \\
\hline Sem informação & 4 & 13,8 \\
\hline Não oferece a disciplina & 1 & 3,4 \\
\hline Primeiro & 1 & 3,4 \\
\hline Segundo & 5 & 17,2 \\
\hline Terceiro & 2 & 6,9 \\
\hline Último & 16 & 55,3 \\
\hline $\begin{array}{l}\text { Proporção da carga horária do curso voltada para a gestão em relação } \\
\text { total do curso }\end{array}$ & \multicolumn{2}{|c|}{ à carga horária } \\
\hline 0 & 6 & 20,8 \\
\hline Até $1 \%$ & 9 & 31,0 \\
\hline 1 a $2 \%$ & 9 & 31,0 \\
\hline Mais de $2 \%$ & 5 & 17,2 \\
\hline
\end{tabular}

Tabela 3. Associação entre as variáveis do estudo e a presença de disciplina específica de gestão, 2019

\begin{tabular}{|c|c|c|c|c|c|}
\hline \multirow{3}{*}{ Variáveis } & \multicolumn{5}{|c|}{ Tem disciplina específica de gestão } \\
\hline & \multicolumn{2}{|c|}{ Sim } & \multicolumn{2}{|c|}{ Não } & \multirow{2}{*}{$\mathbf{p}$} \\
\hline & $\mathbf{n}$ & $\%$ & $\mathbf{n}$ & $\%$ & \\
\hline \multicolumn{6}{|l|}{ Quantidade de horas na graduação } \\
\hline Até 4000 & 4 & 13,8 & 1 & 3,4 & \multirow{4}{*}{0,11} \\
\hline 4001 a 4500 & 7 & 24,1 & 7 & 24,1 & \\
\hline 4501 a 5000 & 2 & 6,9 & 6 & 20,8 & \\
\hline Mais de 5000 & 2 & 6,9 & 0 & 0 & \\
\hline \multicolumn{6}{|l|}{ Natureza da instituição } \\
\hline Particular & 15 & 51,8 & 7 & 24,1 & \multirow{2}{*}{0,09} \\
\hline Pública & 2 & 6,9 & 5 & 17,2 & \\
\hline \multicolumn{6}{|l|}{ Nota Enade } \\
\hline Sem avaliação & 5 & 17,3 & 5 & 17,3 & \multirow{3}{*}{1,02} \\
\hline Até 2 & 3 & 10,3 & 3 & 10,3 & \\
\hline 3 ou mais & 4 & 13,8 & 9 & 31,0 & \\
\hline
\end{tabular}




\section{DISCUSSÃO}

O cadastro das instituições de ensino superior de Odontologia (e-MEC) informa a existência de um total de 380 cursos de Odontologia em atividade no país, excluindo os não iniciados, os extintos e aqueles em fase de extinção. O Paraná responde por 7,6\% dos cursos de Odontologia do país $(\mathrm{n}=29)$.

Foi observado que a maioria das IES no estado do Paraná apresenta disciplina específica de gestão, porém a grande maioria $(51,7 \%)$ dedica menos de $1 \%$ da sua grade horária curricular ao ensino da gestão. Este resultado também foi evidenciado no estudo de Limeira et al. (2018) ${ }^{7}$ que abordou o ensino da gestão no país como um todo. Os autores concluíram que o ensino de gestão é pouco explorado pelos cursos de graduação em Odontologia no Brasil, fazendo-se necessária sua inclusão nas matrizes curriculares, a fim de preparar o cirurgião-dentista para atuar na área da gestão em saúde.

Ceccim e Bilibio (2002) ${ }^{8}$ afirmaram que a formação de recursos humanos possui um vasto conhecimento sobre a relevância da integração ensino-serviço, porém, praticamente inexiste o registro sobre a relevância e necessidade da integração ensino-serviço-gestão-controle social e a articulação com o movimento estudantil de graduação nas profissões da saúde. Os autores afirmam, ainda, que podem ser caracterizadas como inovadoras as experiências integradas entre gestores, formadores, usuários e estudantes, com o objetivo de qualificar a formação dos trabalhadores para as reais necessidades de saúde, e que é necessário que as instituições formadoras realizem importantes iniciativas inovadoras na área do planejamento e da gestão educacionais.

Os profissionais recém-formados na Odontologia, ao entrarem no mercado de trabalho irão se deparar com uma concorrência acirrada. Dados do Conselho Federal de Odontologia (CFO) de $2019^{9}$ demonstram que no estado do Paraná estão registrados 19.969 cirurgiões-dentistas ativos, evidenciando que o mercado de trabalho dos profissionais da área é muito disputado ${ }^{2}$. Dessa forma, a competitividade passa a se constituir em um fator a ser considerado, demandando a formação de profissionais aptos a concorrer às mais diversas funções, para além daquelas inerentes ao atendimento odontológico, mas também envolvendo habilidades de gestão.

Neste estudo foi observado que a maioria $(55,2 \%)$ dos cursos oferta o ensino de gestão nos últimos semestres ou ano de graduação. $\mathrm{O}$ mesmo resultado foi encontrado por Limeira et al. (2018), o que evidencia que os processos de cuidado e gestão não são tratados ou abordados de forma interligada. Assim, primeiramente o estudante aprende a cuidar/tratar do paciente para, só depois, desenvolver uma noção sobre o gerenciamento da assistência em saúde. Isto faz ressaltar a fragmentação do conhecimento, tanto nos diversos processos de ensino quanto na prática das atividades para a gestão, já que a prática, a problematização dos conteúdos e a vivência dos serviços de saúde acontecem em momentos diferentes ${ }^{7}$.

Alguns estudos apontam que um nó crítico da gestão em saúde é o despreparo dos gestores, que fatalmente pecam pela falta de habilidades, competências e atitudes necessárias para uma administração adequada de recursos físicos e humanos $^{7,10,11}$. A falta de profissionalização da gestão foi identificada também no estudo de Lorenzetti et al. (2014) ${ }^{11}$.

Outro ponto essencial, discutido por Limeira et al. $(2018)^{7}$, é a necessidade de pesquisa, formação e inovação no ensino na Área da Saúde, no sentido de viabilizar uma mudança significativa nos modelos de formação profissional que a população e o serviço de saúde brasileiro vigente (Sistema Único de Saúde - SUS) exigem e necessitam. Nesta perspectiva, depreende-se que o gerenciamento desses serviços requer do gestor 
conhecimentos e experiências que os cursos de graduação em Odontologia não estão proporcionando em quantidade e aprofundamento adequados.

O ideal seria que os cursos contemplassem em suas matrizes curriculares o ensino da gestão ao longo do curso, para formar o recurso humano adequado com o perfil recomendado nas DCN: o profissional deve ser capaz de gerenciar e administrar o trabalho, os recursos físicos e materiais e de informação, estando apto a atuar como gestor, empregador ou liderança na equipe de saúde ${ }^{1}$. As DCN citam em seu artigo 11 que a graduação em Odontologia visa à formação do cirurgião-dentista capaz de conhecer, compreender e participar de ações, com vistas à melhoria dos indicadores de qualidade de vida e de morbidade em saúde, passíveis de serem realizados por um profissional generalista, propositivo e resolutivo; desenvolver parcerias, organizar contratos e constituir redes, estimulando e ampliando a aproximação entre instituições, serviços e outros setores envolvidos na atenção integral e promoção da saúde; realizar a gestão do processo de trabalho da equipe de saúde em consonância com o conceito ampliado de saúde e com os princípios e diretrizes do SUS; desenvolver o gerenciamento $\mathrm{e}$ administração da força de trabalho, da informação, dos recursos físicos e materiais; promover a gestão do cuidado, com o uso de saberes e dispositivos de todas as densidades tecnológicas, de modo a promover a organização dos sistemas integrados de saúde para a formulação e desenvolvimento de projetos terapêuticos individuais e coletivos e organizar, manusear e avaliar recursos de cuidados de saúde de forma efetiva e eficiente.

Um fato que chamou atenção durante a consulta ao e-MEC foi a quantidade de cursos novos autorizados e ainda não iniciados no Brasil $(n=88)$ e no estado do Paraná $(n=5)$. Almeja-se que estes cursos estejam mais adequados às DCN, prevendo em seus currículos disciplinas sobre a gestão em saúde do início ao fim do curso. O estudo de Mathias et al. $(2015)^{12}$ mostrou que há excesso de profissionais e de cursos no Brasil, embora com distribuições e características desiguais. O CFO já havia solicitado ao MEC, em novembro de 2017, a suspensão de novos cursos de Odontologia (Ofício CFO 1573/2017) ${ }^{13}$, mas até o momento nenhuma providência foi tomada e por isso refez o pedido no início de 2019. Segundo dados do conselho, atualmente existe um cirurgião-dentista para 645 habitantes no Brasil e o crescimento indiscriminado das instituições que ofertam a graduação em Odontologia pode gerar um colapso na qualidade dos serviços ofertados à população ${ }^{13}$.

$O$ presente estudo permitiu evidenciar que as nomenclaturas normalmente utilizadas nos componentes curriculares que tratam de gestão buscam retratar as temáticas abordadas durante $\mathrm{o}$ curso, uma vez que estes componentes, de um modo geral, procuram contemplar os conhecimentos básicos para a fundamentação teórica do gestor ${ }^{7}$.

Cabe ressaltar algumas limitações deste estudo, primeiramente com relação à coleta de dados secundários, com a análise das matrizes inseridas no endereço eletrônico da IES, que podem ter sua fidedignidade comprometida em função da falta de atualizações constantes. Outro ponto a ser destacado é que alguns cursos não disponibilizavam as matrizes curriculares em seu sítio web, implicando na necessidade de outros estudos, com metodologias diferenciadas que possam complementar as evidências apontadas por esta pesquisa.

Os resultados deste estudo demonstram haver uma grande necessidade em se discutir a formação em gestão pelos cursos de graduação em Odontologia, subsidiando assim a formação de profissionais cada vez mais aptos a contribuírem para a qualificação na gestão do cuidado em saúde.

\section{CONCLUSÃO}

Os resultados sugerem que o ensino de gestão nos cursos de Odontologia nas IES do 
estado do Paraná é pouco explorado, fazendo-se necessária a revisão de abordagens e a destinação de maior carga horária voltada para a gestão em saúde nas matrizes curriculares, a fim de preparar o cirurgião-dentista para atuar nesta área.

\section{ABSTRACT \\ Health management teaching of dentistry courses in Parana}

The study aimed to analyze the teaching of management in dentistry at the universities of the state of Paraná. This was a descriptive, crosssectional research, based on secondary data, conducted in 2019. Information was collected from public and private higher education institutions that offered a dentistry course, based on the list of approved, initiated and postgraduate courses; assets of the national register of higher education courses (e-MEC register). Data were synthesized in a standardized form using Microsoft Excel and after typing, this allowed descriptive analysis by EpiInfo (Version 7.2) and presentation in absolute and relative frequencies. Bivariate analyses were performed by the program Biostat using the $\mathrm{G}$ and Fischer's exact tests, at a significance level of 5\%. In Paraná, 29 Dentistry courses were offered, of which 5 were approved, but had not yet started their activities. Of the researched courses, 21 were of a private (private) administrative nature, seven were public and only one public course that charged the undergraduate students a monthly fee. Most courses $(58.6 \%)$ had a specific management discipline, as a compulsory curriculum component (96.6\%), offered predominantly in the last year of graduation $(55.2 \%)$, with hour loads ranging from 21- to 60 hours (61.9\%). Most $(51.7 \%)$ of undergraduate dentistry courses devote only up to $1 \%$ of their workload to health management. The results showed that management education in this state has been little explored, making it necessary to include a higher workload focused on the theme in the curriculum matrices, aiming to improve the training of future dentists.

Descriptors: Education, Higher. Education, Dental. Health Management Dentistry.

\section{REFERÊNCIAS}

1. Brasil. Ministério da Educação. Conselho Nacional de Educação. Câmara de Educação Superior. Resolução CNE/CES 3, de 19 de fevereiro de 2002. Institui as Diretrizes Curriculares Nacionais do Curso de Graduação em Odontologia. Diário Oficial da União 2002.

2. Ribas MA, Siqueira ES, Binotto E. O desafio da gestão para profissionais da Odontologia. Anais do XXX Encontro Nacional de Engenharia de Produção - ABEPRO, 2010. [Acesso em 14 fev. 2019]. Disponível em: http://www.abepro.org.br/biblioteca/enegep 2010_TN_STO_130_833_15943.pdf.

3. Ceccim RB, Feuerwerker LCM. O Quadrilátero da formação para a Área da Saúde: ensino, gestão, atenção e controle social. Physis: Rev Saúde Col, 2004; 14(1):41-65.

4. Brasil. Ministério da Educação. Cadastro EMEC, regulamentado pela Portaria Normativa ${ }^{\circ} 21$, de 21/12/2017.

5. Programa Epi Info. [Acesso em 14 fev. 2019]. Disponivel em: https://www.cdc.gov/ epiinfo/por/pt_pc.html.

6. Ayres M, Ayres M Jr, Ayres DL.; Santos, A. S. Programa BioEstat. Versão 5.0.3. [Acesso em 14 fev. 2019]. Disponível em: http://www.mamiraua.org.br/download/inde x.php?dirpath=./BioEstat\%205\%20Portugu es\&order $=0 \& g t$.

7. Limeira FIR, Rebouças PRM, Rocha EALS, Catão MHCV. O ensino de gestão nos cursos de graduação em Odontologia no Brasil. Rev ABENO. 2018;18(1):161-9.

8. Ceccim RB, Bilibio LFS. Observação da educação dos profissionais da saúde: evidências à articulação entre gestores, formadores e estudantes. In: BRASIL. Ministério da Saúde. Observatório de recursos humanos em saúde no Brasil: 
estudos e análises. Rio de Janeiro: Fiocruz, 2002.

9. Conselho Federal de Odontologia. [Acesso em 14 fev. 2019]. Disponível em: http://cfo.org.br/website/profissionais-cadas trados/.

10. Okuyama HCHY, Aguilar-da-Silva RH. Gestão do cuidado em Odontologia: limites e potencialidades das ações na Estratégia Saúde da Família. Rev ABENO, 2017;17(4): 133-43.

11. Lorenzetti J, Lanzoni GMM, Assuiti LFC, Pires e Pires EDR, Souza FRS. Gestão em saúde no Brasil: diálogo com gestores públicos e privados. Texto Contexto Enferm. 2014; 23(2): 417-25.
12. Mathias MP, Casania E, Sagazb SM, Lucietto DA. Cirurgiões-dentistas e faculdades no Brasil: repercussões sobre a prática odontológica. J Oral Invest, 2015; $4(2): 25-31$.

13. Conselho Federal de Odontologia. Ofício CFO 1573/2017 que pede a proibição de abertura de novos cursos de Odontologia. 2017. [Acesso em 14 fev. 2019]. Disponível em: http://www.cfo.org.br.

\section{Correspondência para:}

Najara Barbosa da Rocha

e-mail: najara.rocha@gmail.com

Departamento de Odontologia Social e Preventiva, Faculdade de Odontologia, Universidade Federal de Minas Gerais Av. Presidente Antônio Carlos, 6627 - Pampulha 31270-901 Belo Horizonte/MG 